\title{
Combined Inkjet Printing and Infrared Sintering of Silver Nanoparticles using a Swathe-by-Swathe and Layer-by-Layer Approach for 3-Dimensional Structures
}

\author{
Jayasheelan Vaithilingam, ${ }^{* \dagger}{ }^{\dagger}$ Marco Simonelli, ${ }^{\dagger}$ Ehab Saleh, ${ }^{\dagger}$ Nicola Senin, ${ }^{\dagger}$ Ricky D. Wildman, ${ }^{\dagger}$ \\ Richard J. M. Hague, Richard K. Leach, ${ }^{\ddagger}$ and Christopher J. Tuck ${ }^{\dagger}$ \\ ${ }^{\dagger}$ Centre for Additive Manufacturing and ${ }^{\ddagger}$ Manufacturing Metrology Team, Faculty of Engineering, University of Nottingham, \\ Nottingham, U.K., NG7 2RD
}

\section{Supporting Information}

\begin{abstract}
Despite the advancement of additive manufacturing (AM)/3-dimensional (3D) printing, single-step fabrication of multifunctional parts using $A M$ is limited. With the view of enabling multifunctional AM (MFAM), in this study, sintering of metal nanoparticles was performed to obtain conductivity for continuous line inkjet printing of electronics. This was achieved using a bespoke three-dimensional (3D) inkjet-printing machine, JETx, capable of printing a range of materials and utilizing different post processing procedures to print multilayered 3D structures in a single manufacturing step. Multiple layers of silver were printed from an ink containing silver nanoparticles (AgNPs) and infrared sintered using a swathe-by-swathe (SS) and layer-by-layer sintering (LS) regime. The differences in the heat profile for the SS and LS was observed to influence the coalescence

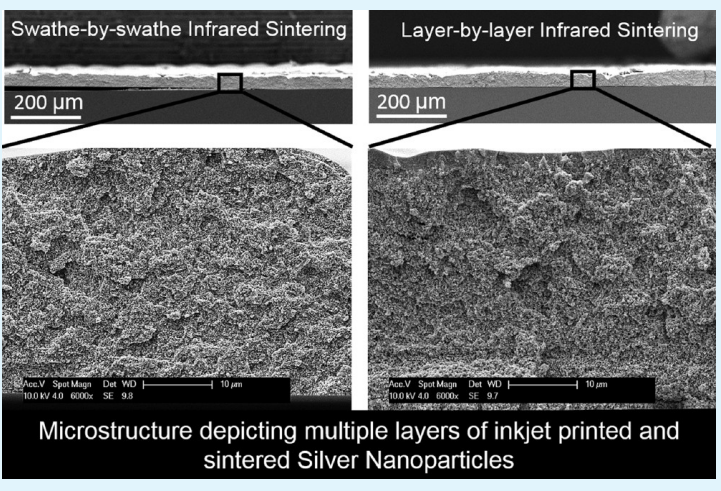
of the AgNPs. Void percentage of both SS and LS samples was higher toward the top layer than the bottom layer due to relatively less IR exposure in the top than the bottom. The results depicted a homogeneous microstructure for LS of AgNPs and showed less deformation compared to the SS. Electrical resistivity of the LS tracks $(13.6 \pm 1 \mu \Omega \mathrm{cm})$ was lower than the SS tracks $(22.5 \pm 1 \mu \Omega \mathrm{cm})$. This study recommends the use of LS method to sinter the AgNPs to obtain a conductive track in $25 \%$ less time than SS method for MFAM.
\end{abstract}

KEYWORDS: silver nanoparticle, 3D-printing, multifunctional additive manufacturing (MFAM), infrared sintering, printed electronics

\section{INTRODUCTION}

Additive manufacturing (AM), also referred as three-dimensional (3D) printing has now evolved from making prototypes to producing end-use parts. Although AM has advanced significantly in recent years, the end-use parts produced by this technology is restricted to single materials in most cases, and limits the disruptive potential of AM to produce parts with multiple functionality. ${ }^{1}$ Multifunctional AM (MFAM) is the fabrication of multifunctional parts using AM technologies and within this context, multifunctionality is the ability of a part to function beyond the structural needs.

Despite the growing interest toward MFAM, the current fabrication of most printed electronics (such as RFID tags, ${ }^{2}$ strain sensors, ${ }^{3}$ humidity sensors, ${ }^{4}$ and passive electronic components ${ }^{5}$ ) is limited to the nano/micron scale in the building $(Z)$ direction, that is, printing less than 20 layers. The studies that demonstrate fully $3 \mathrm{D}$ printed multifunctional structures (millimeters/centimeters in the $\mathrm{Z}$ direction) mostly involve fabrication of parts and components by using extrusion, stereolithography or jetting based printing techniques and embedding an electronic components into it. ${ }^{1,6}$ For example, $\mathrm{Wu}$ et al., $3 \mathrm{D}$ printed parts with a hollow solenoid-shaped channel and injected silver inks to fill the channel to make them conductive for a sensing application. Shemelya et al. ${ }^{8}$ showcased 3D fabrication of sensors by integrating dielectrics with electronic components using a hybrid system. Espalin et al. ${ }^{9}$ fabricated dielectric structures using stereolithography and dispensed conductive inks to form electrical interconnects between components. Despite the advantages of the methods described above to fabricate MFAM components, embedding electrical components and printing conductive tracks, and injecting conductive inks into printed channels will limit the potential benefits that $3 \mathrm{D}$ printing has to offer. Circuits that are traditionally printed in a single plane $(X Y)$ using lithographic techniques can be designed to be printed in multiple planes using $3 \mathrm{D}$ printing, thus opening-up a whole new range of applications. $^{10}$

MFAM, whereby printing multiple materials-both active and passive elements of a part in a single-step may sound simple, but in reality, it is a challenge yet to overcome. Some of the

Received: November 17, 2016

Accepted: January 17, 2017

Published: January 17, 2017 
limiting factors include material availability, printing systems that can print and consolidate multiple materials such as polymers, metals and composites in a single step, and the limited knowledge of printing and consolidating multiple materials to fabricate 3D structures. Also there is a lack of understanding on the interaction between different materials when printed together and ways to incorporate active components into the printed structure to attain multifunctionality. One of the first steps for advancing MFAM is to integrate both multimaterial printing and consolidation steps in situ. A thorough understanding on the printing and consolidation of an active material used for the circuitry is also essential before printing circuits within a $3 \mathrm{D}$ geometry for MFAM.

Material jetting is considered as one of the key enablers of MFAM due to their ability to jet a wide range of materials including polymers, composites and inks containing metal nano particles (MNPs). ${ }^{11-13}$ Ink jet printing (IJP) is a drop-ondemand, noncontact material jetting process capable of direct printing circuits of complex geometries from a computer-aided design (CAD) ${ }^{14}$ In addition to high material utilization rate and lower wastage compared to conventional methods, IJP also has the potential to simplify the manufacturing process of circuits and reduce the associated costs. ${ }^{11}$

In recent years, the use of inks containing MNPs, such as silver, ${ }^{15-18}$ gold, ${ }^{19}$ copper, ${ }^{20}$ and other conductive materials including graphene, ${ }^{21}$ carbon nanotubes, ${ }^{22}$ poly(3.4-ethylenedioxythiophene) polystyrenesulfonate, ${ }^{23}$ and direct writing of liquid metals such as gallium, indium and tin have been widely explored for printing circuits. ${ }^{24-26}$ Although a palette of materials exist for printing conductive tracks, MNPs are still preferred for the manufacturing of circuitry due to lower complexity in the processing of inks containing MNPs and also better resolution and thermal stability compared to conductive polymers and liquid metals. ${ }^{11,27}$ The printed inks containing MNPs are sintered typically at $120-200{ }^{\circ} \mathrm{C}$ depending on the metal, its sintering characteristics, organic ligands and the additives used in the ink formulation. The sintering temperature of the MNPs can be as low as $20 \%$ of the melting temperature of the metal due to their high surface-to-volume ratio. ${ }^{28}$ For example, silver has a melting point of $960{ }^{\circ} \mathrm{C}$ whereas silver nanoparticles (AgNPs) have been observed to sinter at temperatures as low as $150{ }^{\circ} \mathrm{C}$ depending on the particle size. ${ }^{29,30}$ Sintering of MNPs at a low temperature has led to the fabrication of printed flexible electronics on various polymeric substrates such as polyimide. ${ }^{31-33}$ However, most of the commercially available and widely used $3 \mathrm{D}$-inkjet printing platforms (such as Dimatix, ${ }^{29,34}$ Jetlab, ${ }^{11}$ MicroDrop, ${ }^{16}$ Schmid DOD $300,{ }^{35}$ and PiXDRO LP50 ${ }^{36}$ ) are limited to only printing of the inks and are not normally equipped with a method to sinter the MNPs following deposition.

As a consequence, to facilitate the sintering process, the printed pattern is typically transferred to another system equipped with an energy source, such as an oven, ${ }^{27}$ infrared (IR), ${ }^{37}$ electrical, ${ }^{38,39}$ laser, $^{40}$ microwave, ${ }^{41,42}$ and photonic sintering. ${ }^{43}$ Transferring the printed pattern to another system for sintering can be time-consuming and complicated when multiple layers of the same pattern are required to be printed onto each other (i.e., building in the vertical, $Z$ direction). From an industrial point of view, the additional step to sinter the jetted ink can add difficulties for large scale manufacturing and is potentially expensive. These aspects can add further constraints and limit the use of inkjet systems. Thus, there is a requirement for developing a printing system that will suffice the needs of printing and consolidating different materials.

In this study, an ink containing AgNPs was printed using a bespoke 3D multimaterial IJP system, JETx, and subsequently sintered using a built-in IR source. Two distinct sintering methods, swathe-by-swathe (SS) and layer-by-layer (LS) were employed to sinter the AgNP ink. Briefly during SS, each swathe of a layer was sintered using IR whereas during LS, all swathes were printed and subsequently sintered using the IR source. It is envisioned that these sintering mechanism can lead to variation in the layer formation, surface profile, microstructure and the corresponding electrical resistivity of the printed structures. Since the development of multimaterial 3D printing is nascent for electronic applications, it is essential to understand the sintering behavior of AgNPs with these methods. Currently, studies on inkjet printing and sintering of AgNPs are limited to only a few layers (less than 20) and are performed in multiple steps (i.e., printing and sintering on different equipment). In this study, 50 layers of silver were inkjet-printed and sintered in a single step and the impact of SS and LS mechanisms on the printed structure is reported.

\section{RESULTS AND DISCUSSION}

Figure 1 shows the field emission gun-scanning electron microscope (FEG-SEM) micrograph of AgNPs (unsintered)

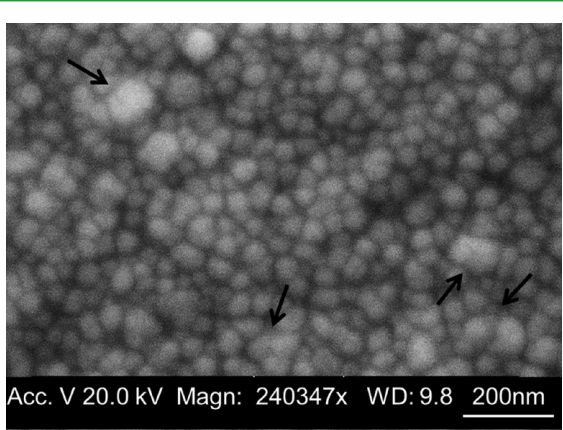

Figure 1. SEM image of the AgNPs present in the ink formulation. The arrows indicate possible agglomeration of the AgNPs.

present in the ink formulation used in this study. It can be seen in Figure 1 that the size of the AgNPs were below $100 \mathrm{~nm}$ and were in agreement with the average size of $\sim 50 \mathrm{~nm}$ quoted by the manufacturer. However, some agglomeration was noted.

Droplet Characterization. Figure 2 shows droplets on a glass (a) and a silver (b) surface (produced by printing a layer of AgNPs to form $20 \mathrm{~mm} \times 20 \mathrm{~mm}$ pattern on a glass slide and IR sintered). The droplets formed on the glass substrate were observed to be smaller $(90 \pm 6 \mu \mathrm{m})$ in size than the droplets formed on the silver surface $(150 \pm 9 \mu \mathrm{m})$. This phenomenon

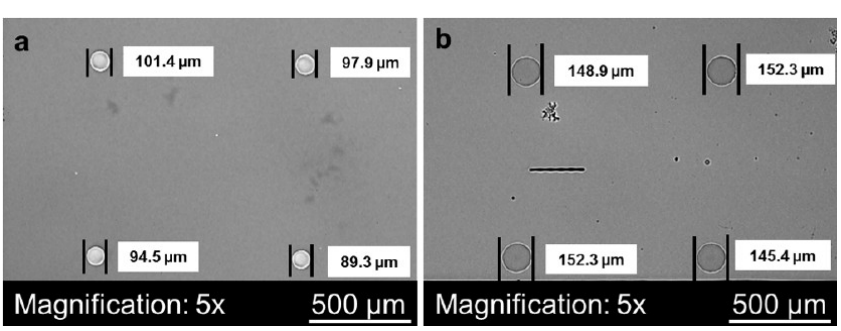

Figure 2. Optical microscopic images showing the droplet size of the silver ink formed on (a) glass substrate and (b) silver surface. 
is mainly due to the different wetting behavior of the silver ink on the glass and the silver surface. ${ }^{34}$

In inkjet printing, the overlapping of droplets is essential to obtain a continuous pattern; however, too much of an overlap can lead to an increased volume of the ink per unit area. If this results in the drop or line exceeding the advancing contact angle, it will lead to the triple line moving beyond the intended location. ${ }^{44}$ Hence drop size is a crucial parameter in calculating the drop overlaps and the size of the features to be printed. Droplet overlap will change depending on the ink, substrate (wettability) and also the surface temperature. As the diameter of the print head nozzle is fixed, the droplet overlap is controlled by increasing or decreasing the resolution of the image (dpi). Since 50 layers of silver were printed in this study, the average drop size obtained for silver on silver $(150 \pm 9 \mu \mathrm{m})$ was used to calculate the drop overlap. The drop diameter obtained for the AgNP ink on the glass surface was insignificant since only one of silver was printed on the glass slide. Several drop overlaps were experimented to maximize the thickness of the layer of silver deposited in one pass. Beyond $65 \%$ overlap, bulging and flow of the ink from the pattern was observed and hence in this study, the drop overlap was limited to $65 \%$.

Surface Morphology. Figure 3 shows the surface morphology of the 50 layers of inkjet printed and sintered

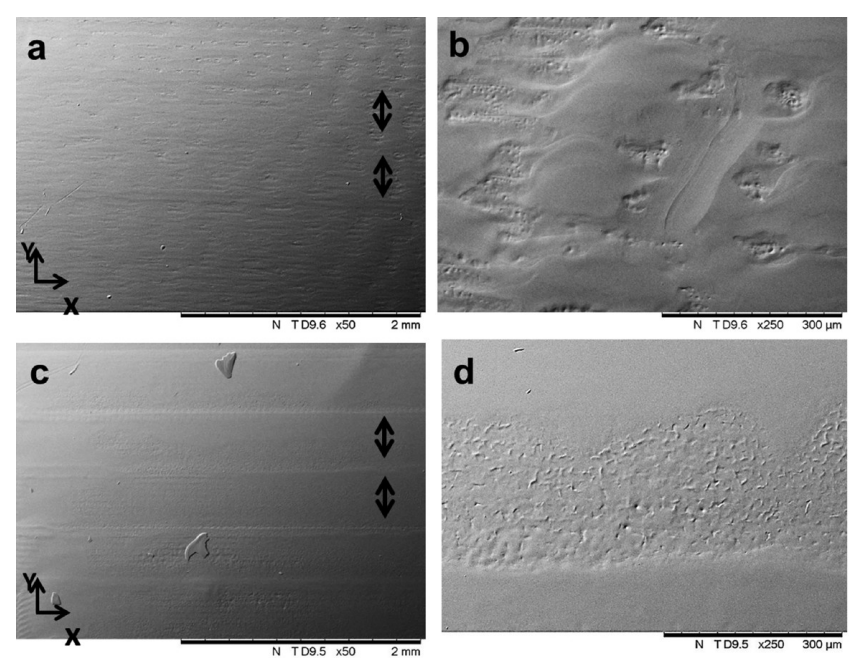

Figure 3. SEM micrographs showing the surface morphology of the inkjet printed and sintered AgNPs. (a) Swathe-by-swathe sintered (SS) silver surface; (b) micropits observed on the SS silver surface; (c) layer-by-layer sintered (LS) silver surface and (d) artifacts observed on the LS surface. The arrow mark indicates the swathes $(\sim 500 \mu \mathrm{m})$.

AgNPs obtained using a benchtop scanning electron microscope (SEM). When using the JETx machine, the $5 \mathrm{~mm} \times 5$ $\mathrm{mm}$ pattern was divided into 10 swathes. The machine prints a swathe using 10 nozzles and hence forming 10 continuous lines/tracks. A regular period at approximately $500 \mu \mathrm{m}$ observed in the figure for both the SS and LS silver pattern (marked with arrows) corresponds to a single swathe. During the SS, each swathe was printed and passed under the IR for sintering before printing the next swathe; whereas during LS, all 10 swathes were printed and then sintered under the IR. As a result, the surface temperature of the printed pattern remained at approximately $138{ }^{\circ} \mathrm{C} \pm 1.5^{\circ} \mathrm{C}$ for SS and $162{ }^{\circ} \mathrm{C} \pm 1.3^{\circ} \mathrm{C}$ for LS. This temperature was recorded using an IR thermometer (error $\pm 2{ }^{\circ} \mathrm{C}$ ) when the substrate reached the home position after the sintering step and before printing the next layer. The IR thermometer was fixed to a tripod to avoid any movement and focused from approximately $15 \mathrm{~cm}$ from the printed surface. These surface temperatures noted for both SS and LS samples exceeded the boiling of the solvent, Triethylene Glycol Monoethyl Ether (TGME; $122^{\circ} \mathrm{C}$ ) present in the ink. As a consequence, the removal of the solvent was likely to be rapid in relation to the possible movement of the triple line and thus pinning the AgNPs to the substrate without movement beyond the desired print location. Hence the individual swathes are visible for both SS and LS samples. However, during SS, since every swathe is passes under the IR, the triple lines are clearly visible than the LS. Micropits were observed on both the SS and LS samples at varying levels. The micropits observed on the SS sample were mostly larger in size than that observed on the LS surface. It should be noted that both surfaces showcased varying levels of roughness and are discussed below.

Surface Topography. The 3D surface topographies of the $5 \mathrm{~mm} \times 5 \mathrm{~mm}$ samples processed by SS and LS methods are shown in Figure $4 \mathrm{a}$ and $4 \mathrm{~b}$. The corresponding twodimensional (2D) profiles, obtained perpendicular to the print direction (along $\mathrm{Y}$ axis) of the SS and LS samples, are shown in Figure $4 \mathrm{c}$ and $4 \mathrm{~d}$. Both the $3 \mathrm{D}$ and the $2 \mathrm{D}$ surface topographies clearly depict the individual swathes $(\sim 500 \mu \mathrm{m}$ each) of the $5 \mathrm{~mm}^{2} \mathrm{SS}$ and LS patterns. The arrow marks in the $3 \mathrm{D}$ profile indicate the areas that were not printed due to clogging of the nozzles. These partially printed locations can also be seen in the $2 \mathrm{D}$ profile (marked with arrows) of the SS and LS surfaces. The double arrows marked in the $2 \mathrm{D}$ profile indicate the profile of a single swathe. Deeper peaks/valleys can be observed toward the edges of the swathes than the center. This is because, during drying, the ink spreads toward the boundaries of the swathes. The flow of the printed ink toward the border is due to capillary flow ${ }^{45}$ (due to the evaporation rate difference between the edge and the center), Marangoni flow $^{46}$ (due to surface tension gradient) and convective flow. ${ }^{47}$ These flows are strongly influenced by the temperature. ${ }^{48}$ In addition to the individual swathes, spreading of ink toward the edges was observed on the overall print pattern leading to slightly thicker edges than at the center of the SS and LS patterns. The thickness of the 50 layers of SS sample was measured to be $33.7 \mu \mathrm{m} \pm 1.6 \mu \mathrm{m}$ and the LS sample thickness was measured as $37.2 \mu \mathrm{m} \pm 1.3 \mu \mathrm{m}$. This result was compared with the thickness obtained for 100 layers for both SS (68.3 $\mu \mathrm{m})$ and LS $(71.3 \mu \mathrm{m})$ samples. The results showed a linear trend with increasing layers.

The arithmetic mean heights $(S a)$ of the SS and LS samples were measured from approximately $2.5 \times 2.5 \mathrm{~mm}$ area of the sample by avoiding the edges due to spreading of the ink to the borders. The $S a$ of the SS sample was $4.53 \mu \mathrm{m}$ whereas for the LS it was 4.82 . The small difference in the $S a$ values between the SS and LS samples were due to the artifacts observed on the surface. Since the surface roughness is an accumulation of the roughness of the printed layers, to verify this phenomena 100 layers of both SS and LS samples were printed. The $S a$ of 100 layers of SS $(8.66 \mu \mathrm{m})$ and LS (9.57) were almost double that of the corresponding 50 layered samples. The deep trench observed due to clogged nozzles is a contributing factors to the increased $S a$ for both samples with increased layers. It should be noted that this study did not attempt to claim which method gives superior profile, but used the profile to observe the spreading of the ink between the two methods. 

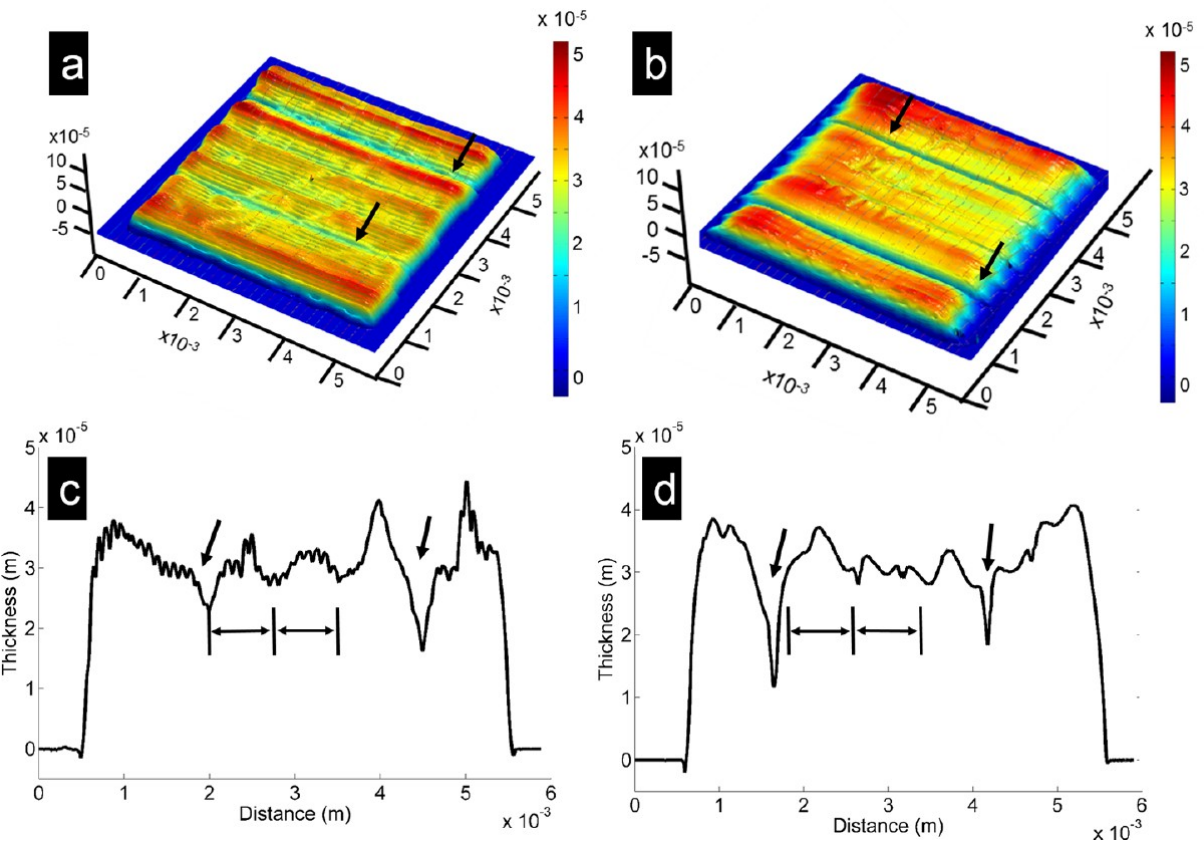

Figure 4. 3D surface topographies of the 50 layers of silver processed using swathe-by-swathe sintered (SS; a) and layer-by-layer sintered (LS; b) and the corresponding 2D surface profiles of the SS (c) and LS (d) samples. Single arrows indicate the missing area due to nozzle failure during printing and double arrows indicate the single print swathe $(\sim 500 \mu \mathrm{m})$ of the SS and LS samples.
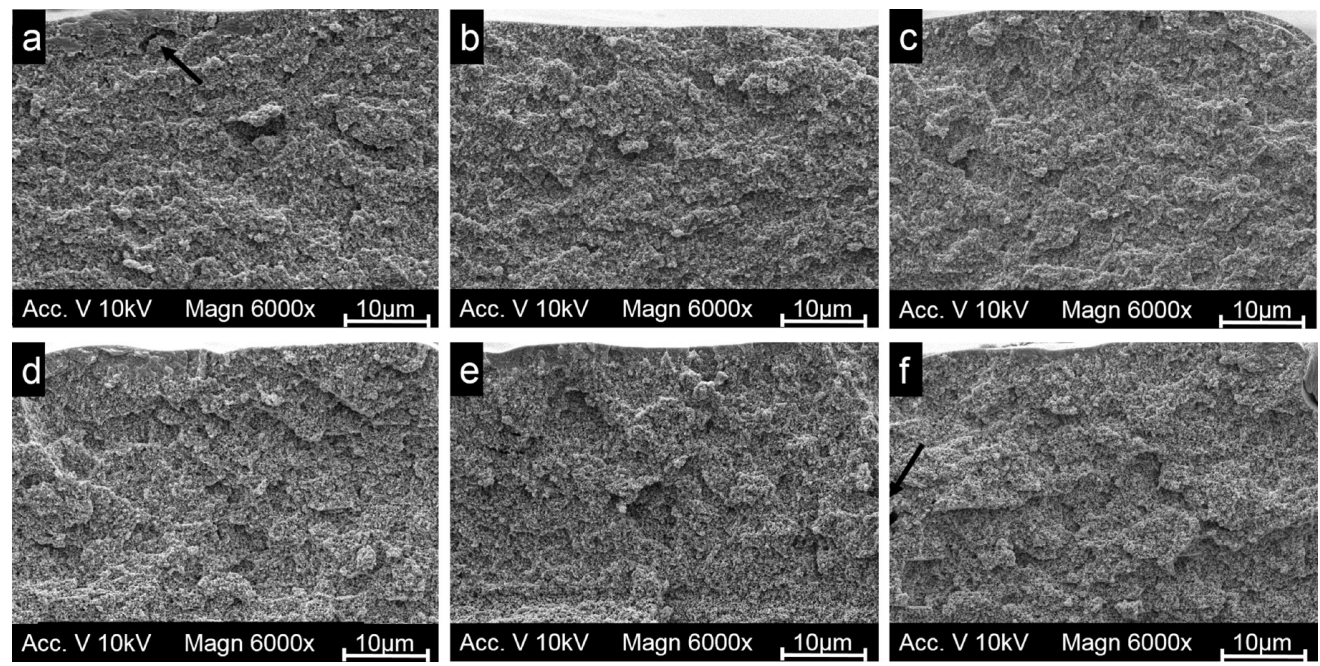

Figure 5. Cross sectioned swathe-by-swathe (SS; a, anterior; b, middle; c, posterior) and layer-by-layer (LS; d, anterior; e, middle; f, posterior) samples depicting their microstructures. Arrows indicate possible voids. The cross sectional images obtained from different locations within the SS and LS sample did not show significant difference between them. Scale bar: $10 \mu \mathrm{m}$.

Microstructure. Initially, to observe the microstructure, the printed 50 layers of the silver on a glass slide was cold mounted in a resin and mechanically polished using a series of grits and abrasive paste. However, the polishing procedure left the samples with a significant number of scratches possibly due to peeling of the sintered silver and glass particles from the mount and the abrasives on the pad. As a result, it was difficult to study the microstructure of the sectioned area. To overcome this issue, the printed SS and LS samples were sectioned perpendicular to the printing direction by gently scoring the printed pattern with a diamond pen and subsequently cracking it. Figure 5 shows the cross section of 50 layers of both SS and LS samples at different areas i.e., anterior, middle and the posterior ends. The cross section of both samples did not depict the existence of macroscopic voids and visually no significant difference was observed for both SS and LL samples.

To examine the microstructures further, the middle region (5th swathe) of the printed pattern was sectioned parallel to the printing direction using a focused ion beam (FIB) and examined using a transmission electron microscope (TEM). The cross sectioned thin films of 3D inkjet-printed SS and LS silver sample, and their corresponding microstructures are shown in Figure 6. The depth of the TEM foil cut from the printed sample was $\sim 5 \mu \mathrm{m}$. Thus, it should be noted that the cross section shown in the Figure $6 \mathrm{a}$ and $6 \mathrm{c}$ is not the cross section of the whole sample. Coalesced AgNPs can be clearly observed from the figure for SS and LS samples. The black areas observed on both SS and LS samples (Figure $6 \mathrm{~b}$ and $6 \mathrm{~d}$ ) 

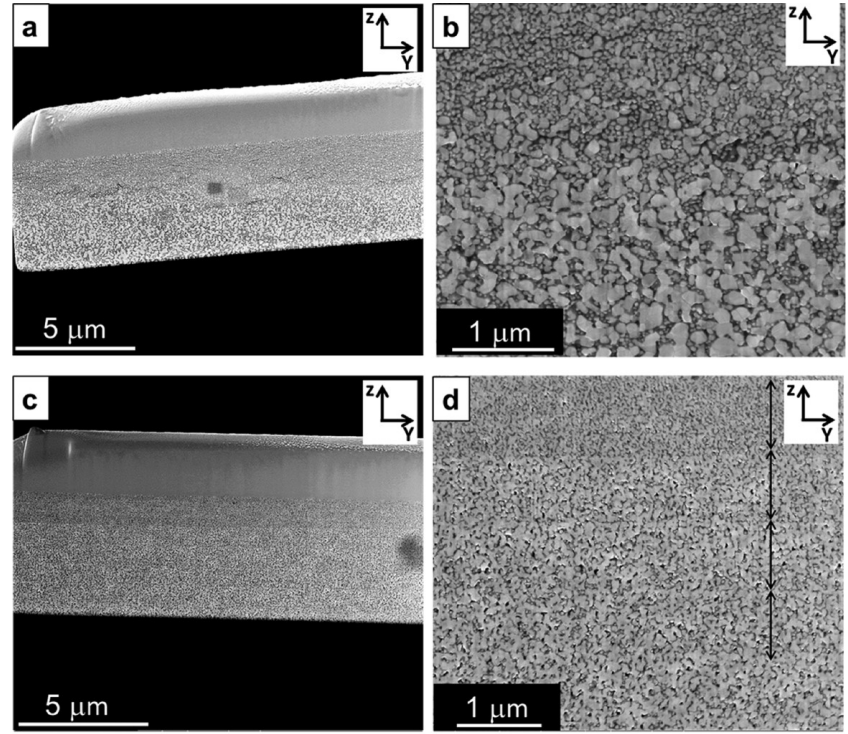

Figure 6. Microstructure of the IR sintered silver nanoparticles (a) cross section of swathe-by-swathe sintered (SS) sample ( top $5 \mu \mathrm{m}$ ); (b) SS sample showing coalescence of AgNPs; (c) cross section of layer-by-layer sintered (LS) sample ( top $6 \mu \mathrm{m}$ ) and (d) coalescence of AgNPs for the LS sample. The double arrows indicate the layer thickness for the LS sample.

are expected to be voids and not solvent residue. This is because during IR sintering, the pattern was exposed to a temperature $\left(>138{ }^{\circ} \mathrm{C}\right)$ higher than the boiling point $\left(122^{\circ} \mathrm{C}\right)$ of the main solvent of the ink, TGME. Similar voids have been previously witnessed for the sintered AgNPs. ${ }^{29,49,50}$ However, existence of residues of the additives from the ink at a very small concentration may be possible. Because of the differences in the milling depth, the cross sectioned LS sample (Figure 6a) appear to be slightly thicker than the SS sample (Figure 6c).

Periodicity in the $Z$ direction was observed for the LS sample at approximately every $800 \mathrm{~nm}$ (marked with an arrow) but not for the SS sample. This periodicity relates to the thickness of a single printed silver layer. One of the possible reasons for SS sample not showing this periodicity could be due to the sintering temperature. As mentioned earlier, since the SS sample moves between the print and the sintering position after every swathe, some of its sample's surface temperature is lost. Consequently during LS, the sample is held under IR for 10 passes continuously. Because the LS sample stays under the IR continuously for 10 exposures, the energy build up is more than the SS. As a result, the residual temperature of LS $\left(\sim 162{ }^{\circ} \mathrm{C}\right)$ was higher than SS $\left(\sim 138^{\circ} \mathrm{C}\right)$ sample and in consequence, the sintered grains showed a varied coalescence behavior for both samples causing it to be difficult to locate the periodicity. The surface profile showed a thickness of $37.2 \pm 1.3 \mu \mathrm{m}$ for the 50 layers of LS sample which equates to approximately $740 \mathrm{~nm}$ per layer. The thickness of the LS sample obtained from the periodicity in the SEM images correlated with the thickness given by the surface profile.

The microstructure of both the SS and LS samples (Figure 6) showed variation in the $Z$ direction. Because of their irregular shape, the size of the coalesced particles was not measured and instead, the void percentage was computed. The SEM images obtained for both the SS and LS samples were processed using MATLAB to obtain the void percentage of the printed patterns. Figure 7 shows the SEM micrograph before and after applying thresholding for SS and LS samples with the graphical representation of the corresponding void percentage. For image processing, white areas were treated as silver and the black areas as voids. Detailed information on the image processing has been given in the experimental methods.

It could be observed from the figure that the void percentage of the SS sample was more than that of the LS sample. Also, it was evident from the graphical representation (Figure $7 \mathrm{c}$ and $7 f$ ) that the SS sample had a significant variation in the void percentage for each segment compared to the LS sample. Since the voids were severe in the top than the bottom, a trend showcasing smaller grains at the top and relatively larger grains at the bottom can be noticed for both the SS and LS samples.
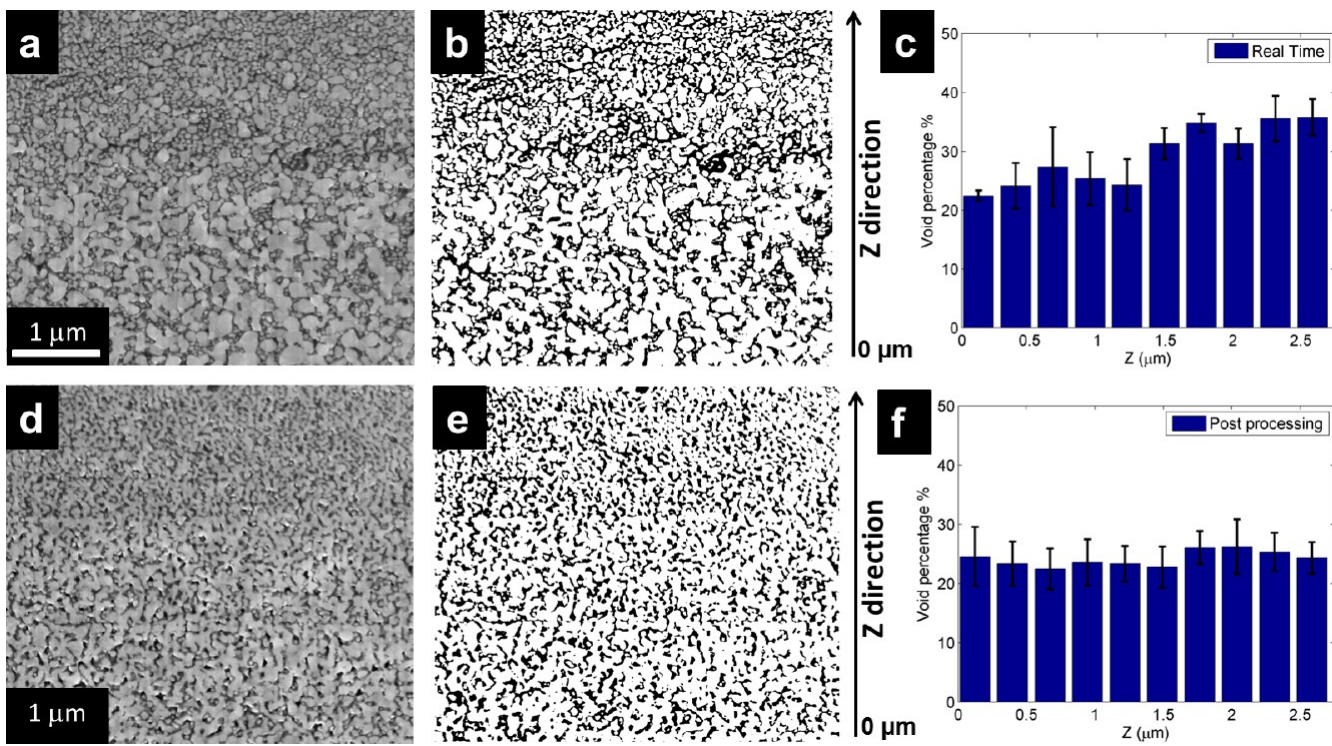

Figure 7. Micrographs used for image processing and the corresponding void percentage: (a) micrograph of a swathe-by-swathe (SS) sample, (b) the corresponding image processed using MATLAB, (c) void percentage of the SS sample [bottom to the top], (d) micrograph of a layer-by-layer (LS) sample, (e) the corresponding image processed using MATLAB, and (f) void percentage of the LS sample [bottom to the top]. 

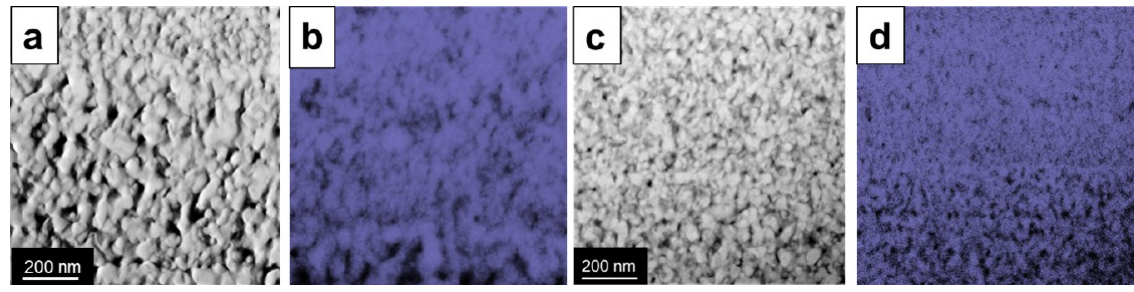

Figure 8. EDX elemental mapping of swathe-by-swathe sintered (SS) and layer-by-layer sintered (LS) sample: (a) SS surface used for elemental mapping, (b) silver (purple) mapped to the SS surface, (c) LS surface used for elemental mapping, and (d) silver (purple) mapped to the LS surface.

As mentioned previously, during SS, every swathe in a layer was scanned under the IR. As a result, the first swathe of every individual layer was subjected to IR sintering 10 times, whereas the last swathe of the same layer was subjected to IR sintering for only once. Also, for this 50 layer build, the first swathe (in the first layer) was heated 500 times and the last swathe (the last swathe of the fiftieth layer) was heated once. Hence the energy supplied to sinter a layer would vary significantly depending on the order of the swathe deposition. Also, the coalescence of the particles usually increases at higher temperature in a thermal sintering process. Thus, as it would be expected, the SS sample showed irregular and a varied coalescence with the top being less and the bottom being more. Similar to the SS sample, the LS sample showed varied coalescence of the AgNPs along the $\mathrm{Z}$ building direction. However, the image processing results revealed a more homogeneous distribution of the coalesced AgNPs for the LS than the SS sample. This is likely due to the higher surface temperature of the LS $\left(162{ }^{\circ} \mathrm{C} \pm 1.3^{\circ} \mathrm{C}\right)$ than the SS $\left(138^{\circ} \mathrm{C}\right.$ $\pm 1.5^{\circ} \mathrm{C}$ ) sample. To confirm this, thermogravimetric analysis (TGA) and differential scanning calorimetry (DSC) analyses were performed for the AgNP ink (Supporting Information). The TGA of the AgNP ink showed a weight loss of $\sim 4 \%$ at 138 ${ }^{\circ} \mathrm{C}$ and $\sim 9 \%$ at $162{ }^{\circ} \mathrm{C}$, revealing rapid solvent evaporation at the surface temperature measured for the LS sample. In line with the TGA results, the DSC also showed a relatively higher heat flow at the sintering temperature of $\operatorname{LS}\left(162^{\circ} \mathrm{C}\right)$ than the SS $\left(138^{\circ} \mathrm{C}\right)$. Hence, the sintering temperature was observed to have a significant effect on the coalescence behavior of the AgNPs.

Despite the nonhomogeneous grain distribution in SS, the coalesced SS grains appear to be larger than the LS. Due to the homogeneous grain structure of the LS (PS) it is difficult to locate the grain boundary and hence it would be difficult to conclude actual grain size of the LS sample. In addition to this, although the SS and LS samples were sectioned in the fifth swathe, it is possible that the imaging was not performed exactly on the same layer. Since both SS and LS showed decrease in grain size with increase in the $Z$ height, slight differences in the location can also contribute to varied grain size. As a result, the grain structure of the SS sample appear to be larger than SS.

One of the disadvantages observed for the SS over LS is the time consumption. The time taken to print and sinter 50 layers of $5 \mathrm{~mm} \times 5 \mathrm{~mm}$ silver pattern was $1 \mathrm{~h}$ for SS and $45 \mathrm{~min}$ for LS method. This is due to the fact that during SS, the substrate moved between print position and sintering position 10 times (to print and sinter every swathe) whereas for LS, the substrate moved once (after printing all swathes). Thus, printing using LS instead of SS can yield a $25 \%$ time reduction overall. Also it should be noted that when printing multiple layers of conductive tracks on a polymeric material, prolonged IR exposure can possibly degrade the material. Hence, the method with less IR exposure time would be ideal.

Chemical Mapping. Figure 8 shows the chemical compositional color mapping of both the SS and LS silver samples. Figure $8 \mathrm{a}$ and $8 \mathrm{c}$ shows the area analyzed for the elemental mapping with an energy dispersive X-rays (EDX) and the corresponding color mapping for silver (Figure $8 b$ and $8 d$ ). The areas of the image rich in purple color represent silver and since the signal intensity dropped, no element was traced on the black areas of the image. As it could be observed from the figure, for both the SS and LS samples, the color mapped images featured primarily silver (in purple). The black areas were predominantly voids due to the absence of material with some signs of carbon residue $(>3 \%)$.

Mechanical Deformation. Deformation of 100 layers of both SS and LS samples for the applied load was studies using an atomic force microscope (AFM) to study the relative strength of the printed samples. Due to the low thickness $(\sim 70$ $\mu \mathrm{m}$ ), the use of other methods such as nanoindendation were not feasible. Figure 9 shows that the deformation of the SS

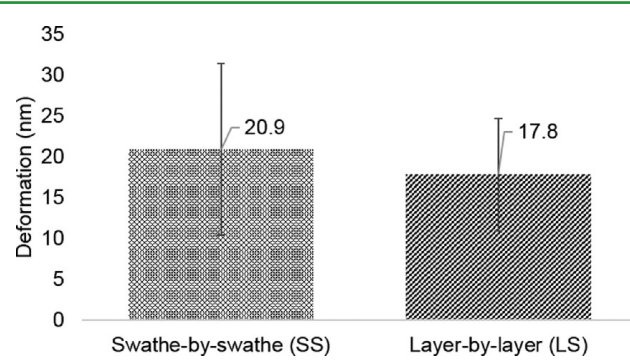

Figure 9. Deformation measured for the swathe-by-swathe sintered (SS) and layer-by-layer sintered (LS) samples for the applied load using an atomic force microscope.

sample was $15 \%$ more than the LS sample. The big deviation noted during the measurement is possibly due to the presence of pores on the surface. Since the voidage of SS was relatively higher than the LS sample in the top few layers (due to less exposure to IR).

Electrical Resistivity. Electrical resistivity of 3D-inkjet printed SS and LS silver tracks was calculated using the relationship $^{51}$

$$
\rho=R\left(\frac{A}{L}\right)
$$

where $\rho$ is the electrical resistivity to be calculated, $R$ is the resistance, $A$ is the cross-sectional area of the track, and $L$ is the length of the track). 50 layers of tracks with $10 \mathrm{~mm}$ length and $1 \mathrm{~mm}$ width were printed and sintered using SS and LS methods. Electrical resistivity of the SS sample $(22.5 \pm 0.1 \mu \Omega$ $\mathrm{cm}$ ) was observed to be higher than that of the LS sample (13.6 
$\pm 0.1 \mu \Omega \mathrm{cm})$. The observed electrical resistivity for the SS and LS samples was nearly 14 and 8 times of the resistivity of bulk silver, respectively. The major reason for the high resistivity of the SS and LS samples compared to the bulk silver is due to the presence of voids in these samples. According to Matthiessen's rule, resistivity is the sum of the resistivities due to temperature, impurities and defects in the metal. There is a percolation threshold above which the material will conduct but the presence of impurities and defects will resist the flow of current. After a certain level (a continuous path is established for the flow of electrons), the presence of voids does not interfere with the conductivity of the material and behave almost similar to the conductivity of a solid material. ${ }^{50}$

Although, the presence of residues from the solvent or other additives in the ink after IR exposure can also be a contributing factor, the elemental analysis picked low amount of carbon (3\%). Hence, voids are expected to be the major factor influencing the resistivity. As expected, due to the presence of more silver/less voids in the LS compared to the SS sample, the resistivity of the LS sample was lower than the SS sample. It has been shown that on increasing the sintering temperature, the electrical resistivity generally decreases. ${ }^{52}$ This is because when the temperature is high, surface energy of the particles would be reduced to form necks through particle boundary and surface diffusion, ${ }^{53}$ thus enabling more contact between the coalesced particles. Since the LS sample remained at relatively high surface temperature $\left(162{ }^{\circ} \mathrm{C} \pm 1.3{ }^{\circ} \mathrm{C}\right)$ compared to the SS sample $\left(138^{\circ} \mathrm{C} \pm 1.5^{\circ} \mathrm{C}\right)$, the resistivity of LS was lower than the SS.

Similar results with resistivity ranging between $2.7 \mu \Omega \mathrm{cm}$ and $90 \mu \Omega \mathrm{cm}$ were witnessed previously for inkjet printed AgNPs. ${ }^{11,32,37,50}$ However, a small difference in the resistivity values between the literature and the current study might have been due to various factors including the substrate used, silver and the additive concentration in the ink, sintering method (such as IR, oven, plasma and laser), time and temperature for sintering, the level of porosity and ink residues in the sintered structure. Resistivity of 10 to 15 times of the bulk silver was previously reported to be sufficient for the printed electronics application $^{50}$ and the resistivity of both LS and SS samples were well within this range.

This method has advantages over the other existing methods since due to the nature of the machine and the process used, multiple materials can be printed and processed in a single step without the need to transfer to other equipment for sintering or further deposition purposes. Since IJP is already capable of printing a range of dielectric and conductive materials, this method will enable users to make multifunctional parts with both conductive and dielectric materials printed in situ in a single step. Also, inkjet printing can precisely position droplets on demand and hence high resolution of printed patterns can be obtained.

Prior studies that report on printing wires, helical structures, bridges, and other 3D structures by using metal nano particles usually requires additional steps such as substrate heating or an annealing/sintering. ${ }^{54,55}$ In view of printing multiple materials, these additional steps will limit contemporaneous printing of multimaterial structures with polymers and conductive metals. The microstructure of printed and sintered AgNPs available in the literature is a limited to less than 20 layers; ${ }^{50,56,57}$ whereas in this study, 50 layers were continuously printed using two sintering methods and their microstructures, surface topography, mechanical strength and their electrical property were evaluated to add to the existing knowledge in the literature. As mentioned earlier, the presented results will be beneficial for enabling the MFAM.

Direct deposition of liquid metals such as gallium, indium, tin, bismuth and their alloys are explored for various electronic applications. ${ }^{24-26}$ Although free-standing structures and high strain rates when stretched can be observed for the printed liquid metals, they have limitations in resolution $(\sim 100 \mu \mathrm{m})$. This is mainly due to the high surface tension of the liquid metal inks such as Ga-In. High precision of the printed patterns required for several complex integrated circuits is difficult to achieve with direct deposition of liquid metals. Also, the resistivity of the tracks printed using the liquid metals is relatively higher than the resistivity offered by the tracks printed using silver and gold nanoparticles. ${ }^{50}$ Adhesion of the printed liquid metals on several substrates are poor and hence, encapsulation of the printed circuitry is a must. ${ }^{58}$ In contrast, relatively better adhesion was observed for the inkjet printed AgNP inks and the printed circuits are normally stable. ${ }^{59}$ In addition, deposition of liquid metals is not compatible with the currently used patterning techniques such as lithography and inkjet printing. The use of parts containing liquid metals are limited to a certain temperature. Formation of sharp edges and connecting the printed electrodes with other materials can be difficult. Since the printed conductive pattern will be in liquid form, under a certain electric/magnetic field, movement of the liquid metal and shifting of the printed pattern is possible which can alternative affect the device performance. ${ }^{58}$ Although the use of liquid metals at a lab scale is demonstrated, scaling-up of this direct deposition of liquid metals may be a challenge.

Despite the advantages of using the proposed method, one has to be aware of the current limitations. The Spectra print head used in the JETx printer has a nozzle diameter of $35 \mu \mathrm{m}$. Hence, the droplet resolution is limited up to $35 \mu \mathrm{m}$. However, the ink's rheology and its spreading behavior plays a crucial role in determining the resolution. IJP depicted coffee-ring effects during solvent drying and hence when building multiple layers, the edges of the printed pattern would be expected to be thicker than the center. Therefore, attaining a uniform layer after each printed layer can be difficult. As mentioned previously, the surface topography and roughness of the final printed layer is the accumulation of every layer. Hence, attaining a uniform layer while printing multiple layers is a challenge. Although inkjet printing enables multimaterial printing, the proposed printing methodology has challenges to overcome. As mentioned above, the print resolution is limited to $35 \mu \mathrm{m}$ with the Spectra SE128 print head used in this study; however, this can be improved by modifying the surface wettability and changing the nozzle size of the print head. The temperature required for sintering AgNPs can be problematic especially when printing on a heat sensitive substrates such as polymers. Since parts are built layer-by-layer, this method to print macroscopic parts with our configuration would be slow. However, the efficiency can be improved by scaling up the number of nozzles and number of print heads. The stability of the ink and nozzle blockage while printing multiple layers could also have a negative impact on the printing process. A combined printing and a cleaning strategy whereby after printing a certain number of layers spitting/purging of the ink can be performed to overcome the nozzle blockage. Obtaining an even surface finish of the inkjet printed parts is typically limited due to an undulating surface. Optimization of process parameters such as print strategy, resolution of the pattern, 
Table 1. Process Conditions Used for SS and LS of AgNPs

\begin{tabular}{|c|c|c|c|c|c|c|}
\hline sintering method & $\begin{array}{l}\text { number of } \\
\text { swathes }\end{array}$ & $\begin{array}{l}\text { printing speed } \\
(\mathrm{mm} / \mathrm{s})\end{array}$ & $\begin{array}{c}\text { IR } \\
\text { intensity } \\
(\%)\end{array}$ & $\begin{array}{l}\text { Height of the substrate } \\
\text { from IR (mm) }\end{array}$ & $\begin{array}{l}\text { rate of substrate movement } \\
\text { under IR }(\mathrm{mm} / \mathrm{s})\end{array}$ & number of passes under IR \\
\hline $\begin{array}{l}\text { swathe-by- } \\
\text { swathe (SS) }\end{array}$ & 10 & 300 & 50 & 5 & 5 & 10 ( 1 pass after every swathe) \\
\hline $\begin{array}{l}\text { layer-by-layer } \\
\text { (LS) }\end{array}$ & 10 & 300 & 50 & 5 & 5 & 10 (after printing a layer) \\
\hline
\end{tabular}

printing conditions, sintering speed and temperature can be performed to obtain a better surface finish. Inevitably, the limited material palette for inkjet printing is another constraint with the use of current method.

\section{CONCLUSION}

This study explored the sintering of inkjet-printed silver nanoparticles using two different IR sintering mechanisms for 3D MFAM. LS of the printed pattern was observed to be superior in terms of the microstructure, mechanical strength and resistivity compared to the SS. Also, fabrication of structures using the LS was $25 \%$ faster compared to the SS. Thus, by inkjet printing and sintering of multiple layers of MNPs in a single step, this study enables the possibilities for the fabrication and realization of 3D-MFAM relatively faster and simpler than the conventional methods.

\section{EXPERIMENTAL METHODS}

Materials. Ink containing silver nanoparticles (Silverjet DGP40LT-15C) was purchased from Advanced Nano Products (ANP). The ink formulation consisted of $38.85 \mathrm{wt} \%$ of silver, $50-60 \mathrm{wt} \%$ of triethylene glycol monoethyl ether (TGME) as solvent and 7-15 wt \% of additives according to the manufacturer's specification. Soda-lime glass slides (Cole-Parmer Instrument Co. Ltd., U.K.) were used to print the patterns. Isopropyl alcohol $\leq 99.5 \%$ for cleaning the glass slide was purchased from Sigma-Aldrich, U.K. A surface mount light emitting diode (LED) was purchased from RS components Ltd., U.K.

Equipment. The inkjet printing machine employed in this study was JETx (Roth \& Rau BV). This bespoke machine is built with six print heads (Spectra SE128). The build volume (XYZ) of the machine is $200 \mathrm{~mm} \times 200 \mathrm{~mm} \times 190 \mathrm{~mm}$. The machine is equipped with an ultraviolet (UV) radiation source with a wavelength of $395 \mathrm{~nm}$ and an output of $2 \mathrm{~W} / \mathrm{cm}^{2}$, and an infrared lamp $(1 \mathrm{~kW})$ with a maximum filament temperature of $1500{ }^{\circ} \mathrm{C}$ for curing/sintering of heat/ photosensitive materials. More details have been provided in the Supporting Information. An RS $1327 \mathrm{~K}$ infrared thermometer (RS components Ltd., U.K.; accuracy $\pm 2{ }^{\circ} \mathrm{C}$ ) was used to measure the surface temperature of the printed pattern.

Design. A $5 \mathrm{~mm} \times 5 \mathrm{~mm}$ pattern with 500 dpi was designed using the open source software, GIMP (The General Image Manipulation Program). The file was saved as bitmap (.bmp) image file format. Three tracks each with the dimensions $20 \mathrm{~mm} \times 1 \mathrm{~mm}$ and $500 \mathrm{dpi}$ was designed for electrical resistance measurement. A bitmap file (500 dpi) showcasing one pixel at regular intervals $(1 \mathrm{~mm})$ for drop size characterization was also designed using GIMP.

3D-Printing. The glass slides used to build the pattern were rinsed with isopropyl alcohol and dried to remove contaminants from atmosphere. The build plate of the JETx machine is typically maintained at $20{ }^{\circ} \mathrm{C}$ to prevent the deformation of the platform because of thermal expansion. This chilled platform will counteract with the heat produced by the IR and affect the sintering of silver. Hence the glass slide was positioned above two ceramic chalks to raise it $5 \mathrm{~mm}$ above the build platform and have air as insulator between the slide and the build platform. A material file defining the process parameters was created using the inbuilt JETx recipe generator. The previously optimized process parameters were used in this study and they are tabulated in Table 1. Once the material file is applied, printing was started. Briefly during printing, the build platform moved in $X$ direction and the print heads moved in $Y$ and $Z$ direction. After printing a swath or a layer (depending on SS or LS), the build platform moved under the IR lamp and the AgNPs were sintered under the specified conditions. Once sintering of the swath (SS) is complete, the platform moved back to the printing station for the next print. On completing a layer, the build platform returned to home position and then moved to the print station. This continues until the whole pattern (50 layers) was printed. For LS sample, every layer was printed and subsequently passed under the IR for sintering. Finally the glass slide with the inkjet-printed and sintered 50 layers of silver was removed from the platform and characterized. Similar procedure was used to print and sinter 50 layers of tracks for electrical resistivity measurement and the demonstrator. The surface temperature of the SS and LS sample after printing and sintering every layer of the $5 \mathrm{~mm}$ $\times 5 \mathrm{~mm}$ pattern was measured using an IR thermometer fixed next to the print platform and averaged. It should be noted that the printed pattern for the droplet characterization was not sintered.

Droplet Characterization. The single droplets printed on the glass slide and on silver coated glass slide were observed under Nikon Eclipse (LV100ND) optical microscope. Using in-built software, the droplet size was measured. The size of 15 droplets were measured and averaged.

Sample Preparation. To examine the cross sectional area of the printed sample, the printed samples on a glass slide scored perpendicular to the print direction using a diamond pen and gently broken. One of the two broken slide was cold mounted using a mixture $(7.5 \mathrm{~mL}: 1 \mathrm{~mL})$ of an epoxy resin and a hardener. The mounted sample was mechanical polishing was performed using silicon carbide grits (P280, P400, P800, and P1200) and diamond paste (6 and $1 \mu \mathrm{m}$ ) supplied by Buehler, U.K. For force curve measurement using atomic force microscope, 100 layers SS and LS samples printed on a glass slide was cold mounted using an epoxy resin. The samples were mounted such that the top surface of the sample can be used for the study. The mounted sample was gently ground using a P1200 grit to remove polymer residues and polished using polishing cloth with 6 and $1 \mu \mathrm{m}$ diamond paste as the abrasive. Polishing was gently performed such that the printed layers were not completely removed. Polishing was necessary to remove the wavy profile of the surface and aid precise measurement for AFM tip. The polished surface was examined using the optical microscope before AFM characterization.

Surface Characterization. Surface Topography Measurement. The surface topography of the printed silver tracks was obtained using focus-variation 3D microscope (Alicona Infinite Focus G5), ${ }^{\circ}$ equipped with a $10 \times$ objective with pixel size, $0.88 \mu \mathrm{m} \times 0.88 \mu \mathrm{m}$; size of an individual field-of-view (FOV), approximately $1.62 \mathrm{~mm} \times$ $1.62 \mathrm{~mm}(1840 \times 1840$ pixels $)$. Polarized light was used to compensate for the high reflectivity of the track surfaces. Individual tracks were coarsely aligned to the instrument $x$-axis and measured in stitching mode (up to $6 \times 1$ stitched, individual FOVs), typically covering 3-6 $\mathrm{mm}$ of track, depending on track characteristics. A dedicated procedure for dimensional and geometric characterization of the individual tracks was devised based on previous work. ${ }^{61}$ For computing the $S a$ parameter, the topographies were subjected to leveling by least-squares mean plane subtraction, with no additional filtering operation. Arithmetic mean height $(\mathrm{Sa})$ was obtained from an area of approximately $2.5 \mathrm{~mm} \times 2.5 \mathrm{~mm}$ avoiding the edges. The $2 \mathrm{D}$ surface profile was obtained vertically $(Y)$ from the center of the pattern. Further information detailing surface topography data processing and analysis for an individual track is given in the Supporting Information. 
Surface Morphology. Nikon Eclipse (LV100ND) optical microscope was used to obtain the surface morphology of the SS and LS silver patterns $\left(5 \mathrm{~mm}^{2}\right)$. Surface morphology of the printed SS and LS patterns was obtained using a Hitachi TM3030 tabletop scanning electron microscope (SEM). Acceleration voltage of $15 \mathrm{kV}$ was used and the images were acquired in the topography mode.

Microstructure. FEI XL30 (Philips) scanning electron microscope (SEM) fitted with a tungsten hairpin filament was used to obtain the microstructure of the cross sectional area of SS and LS samples. To study the microstructure in detail, transmission electron microscope (TEM; TECNAI F20 TEM) was employed. Electron transparent foil from the SS and LS samples were initially prepared using a focused ion beam (FIB)-SEM workstation (Nova 600 Nanolab Dual Beam). Bright-field imaging and EDX mapping were performed using an accelerating voltage of $200 \mathrm{kV}$. The $5 \mathrm{~mm}^{2}$ pattern was divided into 10 swathes and hence, the microstructural analysis was performed on the middle of the printed pattern, that is, in the fifth swathe.

Image Processing. SEM micrographs of the SS and LS samples were image processed in MATLAB. The SEM images used for the image processing were obtained from the top regions of the 50 layer SS and LS samples. Various thresholding values were used and the thresholded images were superimposed on the original image until a true representation of the image was obtained. The thresholding value for the SS and LS images were same. The SS and LS images were then sectioned into 10 equal segments in the building $Z$ direction and 5 equal segments in the $X$ direction to calculate the void percentage as a function of building direction. The sectioned images were analyzed for the black versus white areas. The black pixels were accounted as voids and the white pixels as silver. The obtained mean void percentage was plotted graphically and the reported standard deviation was obtained from the 5 segments of the image along the $X$ direction.

Mechanical Deformation. D3000 Bruker Atomic force microscope (AFM) was used to study the deformation behavior of the printed samples. Bruker MPP-13100-10 rectangular tip with a resonant frequency of $525 \mathrm{kHz}$ and a spring constant of $200 \mathrm{~N} / \mathrm{m}$ was employed in this study. No modification to the tip was performed to obtain the mechanical force curve. Force measurement was performed in contact-mode. Detector sensitivity was calibrated based on the force curve obtained for the plain glass used to print the samples. 100 measurement were performed for both samples on an area away from the edges and trenches.

Electrical Resistivity. Electrical resistivity for SS and LS was obtained by measuring the resistance offered by the 50 layered tracks using a Hameg LCR high precision meter (HM 8018). The volume of the tracks were computed by integrating the surface profiles of the tracks obtained from surface profilometry. The possible error of \pm 1 $\mu \Omega$ due to the LCR equipment should be accounted. The reported values in this study are an average of the electrical resistivity of three different tracks.

\section{ASSOCIATED CONTENT}

\section{S Supporting Information}

The Supporting Information is available free of charge on the ACS Publications website at DOI: 10.1021/acsami.6b14787.

Description of JETx 3D multimaterial inkjet printing system, thermal analysis of silver nanoparticles using differential scanning calorimetry and thermogravimetry, and methodology employed for the processing and analysis of surface topography data (PDF)

\section{AUTHOR INFORMATION}

\section{Corresponding Author}

*E-mail: Jayasheelan.Vaithilingam@nottingham.ac.uk. Tel: +44 (0)115 82 32334. Fax: +44 (0)11595 13948.

ORCID

Jayasheelan Vaithilingam: 0000-0002-5501-5344

\section{Notes}

The authors declare no competing financial interest.

\section{ACKNOWLEDGMENTS}

The authors acknowledge the assistance of Prof. Chen Xinyong for the support towards AFM characterization and Nanoscale and Microscale Research Centre (NMRC) at the University of Nottingham for SEM access. This work was supported by the Engineering and Physical Sciences Research Council (grant number EP/I033335/2). The authors, Nicola Senin and Richard K Leach would also like to acknowledge the funding support from the EC grant METROSURF (FP7-PEOPLEIEF).

\section{REFERENCES}

(1) MacDonald, E.; Wicker, R. Multiprocess 3D Printing for Increasing Component Functionality. Science 2016, 353, aaf2093.

(2) Vyas, R.; Lakafosis, V.; Lee, H.; Shaker, G.; Yang, L.; Orecchini, G.; Traille, A.; Tentzeris, M. M.; Roselli, L. Inkjet Printed, Self Powered, Wireless Sensors for Environmental, Gas, and Authentication-Based Sensing. IEEE Sens. J. 2011, 11, 3139-3152.

(3) Muth, J. T.; Vogt, D. M.; Truby, R. L.; Mengüç, Y.; Kolesky, D. B.; Wood, R. J.; Lewis, J. A. Embedded 3D Printing of Strain Sensors within Highly Stretchable Elastomers. Adv. Mater. 2014, 26, 63076312.

(4) Leigh, S. J.; Bradley, R. J.; Purssell, C. P.; Billson, D. R.; Hutchins, D. a. A Simple, Low-Cost Conductive Composite Material for 3D Printing of Electronic Sensors. PLoS One 2012, 7, e49365.

(5) Kang, B. J.; Lee, C. K.; Oh, J. H. All-Inkjet-Printed Electrical Components and Circuit Fabrication on a Plastic Substrate. Microelectron. Eng. 2012, 97, 251-254.

(6) Kim, H. S.; Kang, J. S.; Park, J. S.; Hahn, H. T.; Jung, H. C.; Joung, J. W. Inkjet Printed Electronics for Multifunctional Composite Structure. Compos. Sci. Technol. 2009, 69, 1256-1264.

(7) Wu, S.-Y.; Yang, C.; Hsu, W.; Lin, L. 3D-Printed Microelectronics for Integrated Circuitry and Passive Wireless Sensors. Microsystems Nanoeng. 2015, 1, 15013.

(8) Shemelya, C.; Banuelos-chacon, L.; Melendez, A.; Kief, C.; Espalin, D.; Wicker, R; et al. Multi-Functional 3D Printed and Embedded Sensors for Satellite Qualification Structures. Sensors 2015, $1-4$.

(9) Espalin, D.; Muse, D. W.; MacDonald, E.; Wicker, R. B. 3D Printing Multifunctionality: Structures with Electronics. Int. J. Adv. Manuf. Technol. 2014, 72, 963-978.

(10) Hart, L. R.; Li, S.; Sturgess, C.; Wildman, R.; Jones, J. R.; Hayes, W. 3D Printing of Biocompatible Supramolecular Polymers and Their Composites. ACS Appl. Mater. Interfaces 2016, 8, 3115-3122.

(11) Yin, Z. P.; Huang, Y. a.; Bu, N. B.; Wang, X. M.; Xiong, Y. L. Inkjet Printing for Flexible Electronics: Materials, Processes and Equipments. Chin. Sci. Bull. 2010, 55, 3383-3407.

(12) Zhang, F.; Tuck, C.; Hague, R.; He, Y.; Saleh, E.; Li, Y.; Sturgess, C.; Wildman, R. Inkjet Printing of Polyimide Insulators for the 3D Printing of Dielectric Materials for Microelectronic Applications. J. Appl. Polym. Sci. 2016, 133, 43361.

(13) He, Y.; Wildman, R. D.; Tuck, C. J.; Christie, S. D. R.; Edmondson, S. An Investigation of the Behavior of Solvent Based Polycaprolactone Ink for Material Jetting. Sci. Rep. 2016, 6, 20852.

(14) Chen, X.; Ashcroft, I. A.; Wildman, R. D.; Tuck, C. J. An Inverse Method for Determining the Spatially Resolved Properties of Viscoelastic - Viscoplastic Three-Dimensional Printed Materials. Proc. R. Soc. London, Ser. A 2015, 471, 20150477.

(15) Walker, S. B.; Lewis, J. A. Reactive Silver Inks for Patterning High-Conductivity Features at Mild Temperatures. J. Am. Chem. Soc. 2012, 134, 1419-1421.

(16) Futera, K.; Kielbasinski, K.; Młozniak, A.; Jakubowska, M. Inkjet Printed Microwave Circuits on Flexible Substrates Using Heterophase 
Graphene Based Inks. Soldering Surf. Mount Technol. 2015, 27, 112114.

(17) Chen, S.-P.; Chiu, H.-L.; Wang, P.-H.; Liao, Y.-C. Inkjet Printed Conductive Tracks for Printed Electronics. ECS J. Solid State Sci. Technol. 2015, 4, P3026-P3033.

(18) Bidoki, S. M.; Lewis, D. M.; Clark, M.; Vakorov, a; Millner, P. a; McGorman, D. Ink-Jet Fabrication of Electronic Components. J. Micromech. Microeng. 2007, 17, 967-974.

(19) Määttänen, A.; Ihalainen, P.; Pulkkinen, P.; Wang, S.; Tenhu, H.; Peltonen, J. Inkjet-Printed Gold Electrodes on Paper: Characterization and Functionalization. ACS Appl. Mater. Interfaces 2012, 4, 955-964.

(20) Jang, S.; Seo, Y.; Choi, J.; Kim, T.; Cho, J.; Kim, S.; Kim, D. Sintering of Inkjet Printed Copper Nanoparticles for Flexible Electronics. Scr. Mater. 2010, 62, 258-261.

(21) Torrisi, F.; Hasan, T.; Wu, W.; Sun, Z.; Lombardo, A.; Kulmala, T. S.; Hsieh, G. W.; Jung, S.; Bonaccorso, F.; Paul, P. J.; et al. InkjetPrinted Graphene Electronics. ACS Nano 2012, 6, 2992-3006.

(22) Wang, T.; Roberts, M. a; Kinloch, I. a.; Derby, B. Inkjet Printed Carbon Nanotube Networks: The Influence of Drop Spacing and Drying on Electrical Properties. J. Phys. D: Appl. Phys. 2012, 45, 315304.

(23) Perinka, N.; Kim, C. H.; Kaplanova, M.; Bonnassieux, Y. Preparation and Characterization of Thin Conductive Polymer Films on the Base of PEDOT:PSS by Ink-Jet Printing. Phys. Procedia 2013, 44, 120-129.

(24) Ladd, C.; So, J. H.; Muth, J.; Dickey, M. D. 3D Printing of Free Standing Liquid Metal Microstructures. Adv. Mater. 2013, 25, 50815085 .

(25) Joshipura, I. D.; Ayers, H. R.; Majidi, C.; Dickey, M. D. Methods to Pattern Liquid Metals. J. Mater. Chem. C 2015, 3, 3834-3841.

(26) Wang, L.; Liu, J. Liquid Phase 3D Printing for Quickly Manufacturing Conductive Metal Objects with Low Melting Point Alloy Ink. Sci. China: Technol. Sci. 2014, 57, 1721-1728.

(27) Kamyshny, A.; Steinke, J.; Magdassi, S. Metal-Based Inkjet Inks for Printed Electronics. Open Appl. Phys. J. 2011, 4, 19-36.

(28) Hummelgard, M.; Zhang, R; Nilsson, H.-E.; Olin, H. Electrical Sintering of Silver Nanoparticle Ink Studied by In-Situ TEM Probing. PLoS One 2011, 6, e17209.

(29) Molina-Lopez, F.; Briand, D.; De Rooij, N. F. All Additive Inkjet Printed Humidity Sensors on Plastic Substrate. Sens. Actuators, B 2012, 166-167, 212-222.

(30) Magdassi, S.; Grouchko, M.; Berezin, O.; Kamyshny, A. Triggering the Sintering of Silver Nanoparticles at Room Temperature. ACS Nano 2010, 4, 1943-1948.

(31) Bonea, A.; Brodeala, A.; Vlǎdescu, M.; Svasta, P. Electrical Conductivity of Inkjet Printed Silver Tracks. Proc. Int. Spring Semin. Electron. Technol. 2012, 1-4.

(32) Kang, J. S.; Ryu, J.; Kim, H. S.; Hahn, H. T. Sintering of InkjetPrinted Silver Nanoparticles at Room Temperature Using Intense Pulsed Light. J. Electron. Mater. 2011, 40, 2268-2277.

(33) Yoon, S. H.; Lee, J. H.; Lee, P. C.; Nam, J. Do; Jung, H.-C.; Oh, Y. S.; Kim, T. S.; Lee, Y. K. Sintering and Consolidation of Silver Nanoparticles Printed on Polyimide Substrate Films. Macromol. Res. 2009, 17, 568-574.

(34) Lee, S.-H.; Cho, Y.-J. Characterization of Silver Inkjet OverlapPrinting through Cohesion and Adhesion. J. Electr. Eng. Technol. 2012, 7, 91-96.

(35) Polzinger, B.; Schoen, F.; Matic, V.; Keck, J.; Willeck, H.; Eberhardt, W.; Kueck, H. UV-Sintering of Inkjet-Printed Conductive Silver Tracks. 2011 11th IEEE Int. Conf. Nanotechnol. 2011, 201-204.

(36) Bram, M.; Dornseiffer, J.; Hoffmann, J.; van Gestel, T.; Meulenberg, W. a.; Stöver, D. Inkjet Printing of Microporous Silica Gas Separation Membranes. J. Am. Ceram. Soc. 2015, 98, 2388-2394.

(37) Tobjörk, D.; Aarnio, H.; Pulkkinen, P.; Bollström, R.; Määttänen, A.; Ihalainen, P.; Mäkelä, T.; Peltonen, J.; Toivakka, M.; Tenhu, H.; et al. IR-Sintering of Ink-Jet Printed Metal-Nanoparticles on Paper. Thin Solid Films 2012, 520, 2949-2955.
(38) Roberson, D. A.; Wicker, R. B.; MacDonald, E. Ohmic Curing of Printed Silver Conductive Traces. J. Electron. Mater. 2012, 41, 25532566.

(39) Roberson, D. a.; Wicker, R. B.; MacDonald, E. Ohmic Curing of Three-Dimensional Printed Silver Interconnects for Structural Electronics. J. Electron. Packag. 2015, 137, 031004.

(40) Lopes, A. J.; Lee, I. H.; Macdonald, E.; Quintana, R.; Wicker, R. Laser Curing of Silver-Based Conductive Inks for in Situ 3D Structural Electronics Fabrication in Stereolithography. J. Mater. Process. Technol. 2014, 214, 1935-1945.

(41) Perelaer, J.; De Gans, B. J.; Schubert, U. S. Ink-Jet Printing and Microwave Sintering of Conductive Silver Tracks. Adv. Mater. 2006, $18,2101-2104$.

(42) Perelaer, J.; Klokkenburg, M.; Hendriks, C. E.; Schubert, U. S. Microwave Flash Sintering of Inkjet-Printed Silver Tracks on Polymer Substrates. Adv. Mater. 2009, 21, 4830-4834.

(43) West, J.; Carter, M.; Smith, S.; Sears, J. Photonic Sintering of Silver Nanoparticles: Comparison of Experiment and Theory. Sinter. Methods Prod. 2012, 173-189.

(44) Soltman, D.; Subramanian, V. Inkjet-Printed Line Morphologies and Temperature Control of the Coffee Ring Effect. Langmuir 2008, 24, 2224-2231.

(45) Deegan, R. D.; Bakajin, O.; Dupont, T. F.; Huber, G.; Nagel, S. R; Witten, T. a. Capillary Flow as the Cause of Ring Stains from Dried Liquid Drops. Nature 1997, 389, 827-829.

(46) Kim, D.; Jeong, S.; Shin, H.; Xia, Y.; Moon, J. Heterogeneous Interfacial Properties of Ink-Jet-Printed Silver Nanoparticulate Electrode and Organic Semiconductor. Adv. Mater. 2008, 20, 30843089.

(47) Zhou, W.; List, F. a.; Duty, C. E.; Babu, S. S. Sintering Kinetics of Inkjet-Printed Conductive Silver Lines on Insulating Plastic Substrate. Metall. Mater. Trans. B 2015, 46, 1542-1547.

(48) Zheng, Y.; Li, S.; Shi, W.; Yu, J. Spray-Coated Nanoscale Conductive Patterns Based on in Situ Sintered Silver Nanoparticle Inks. Nanoscale Res. Lett. 2014, 9, 145.

(49) Allen, M. L.; Aronniemi, M.; Mattila, T.; Alastalo, A.; Ojanperä, K.; Suhonen, M.; Seppä, H. Electrical Sintering of Nanoparticle Structures. Nanotechnology 2008, 19, 175201.

(50) Niittynen, J.; Abbel, R.; Mäntysalo, M.; Perelaer, J.; Schubert, U. S.; Lupo, D. Alternative Sintering Methods Compared to Conventional Thermal Sintering for Inkjet Printed Silver Nanoparticle Ink. Thin Solid Films 2014, 556, 452-459.

(51) Kamyshny, A.; Magdassi, S. Conductive Nanomaterials for Printed Electronics. Small 2014, 10, 3515-3535.

(52) Huang, Q.; Shen, W.; Xu, Q.; Tan, R.; Song, W. Properties of Polyacrylic Acid-Coated Silver Nanoparticle Ink for Inkjet Printing Conductive Tracks on Paper with High Conductivity. Mater. Chem. Phys. 2014, 147, 550-556.

(53) Alarifi, H. A.; Atis, M.; Ozdogan, C.; Hu, A.; Yavuz, M.; Zhou, Y. Molecular Dynamics Simulation of Sintering and Surface Premelting of Silver Nanoparticles. Mater. Trans. 2013, 54, 884-889.

(54) An, B. W.; Kim, K.; Lee, H.; Kim, S. Y.; Shim, Y.; Lee, D. Y.; Song, J. Y.; Park, J. U. High-Resolution Printing of 3D Structures Using an Electrohydrodynamic Inkjet with Multiple Functional Inks. Adv. Mater. 2015, 27, 4322-4328.

(55) Sadie, J. A.; Subramanian, V. Three-Dimensional Inkjet-Printed Interconnects Using Functional Metallic Nanoparticle Inks. Adv. Funct. Mater. 2014, 24, 6834-6842.

(56) Denneulin, A.; Blayo, A.; Neuman, C.; Bras, J. Infra-Red Assisted Sintering of Inkjet Printed Silver Tracks on Paper Substrates. J. Nanopart. Res. 2011, 13, 3815-3823.

(57) Haque, R.; Ogam, E.; Loussert, C.; Benaben, P.; Boddaert, X. Fabrication of Capacitive Acoustic Resonators Combining 3D Printing and 2D Inkjet Printing Techniques. Sensors 2015, 15, 26018-26038.

(58) Wang, X.; Liu, J. Recent Advancements in Liquid Metal Flexible Printed Electronics: Properties, Technologies and Applications. micromachines 2016, 7, 206. 
(59) Joo, S.; Baldwin, D. F. Adhesion Mechanisms of Nanoparticle Silver to Substrate Materials: Identification. Nanotechnology 2010, 21, 055204.

(60) Helmli, F. Focus Variation Instruments. In Optical Measurement of Surface Topography; Leach, Ri. K., Ed.; Springer Berlin Heidelberg: Berlin, 2011; pp 131-166.

(61) Senin, N.; Blunt, L. Characterisation of Individual Areal Features, in Characterisation of Areal Surface Texture. In Characterisation of Arial Surface Texture; Leach, R., Ed.; Springer: Heidelberg, 2013; pp 179-216. 